\title{
Crosstalk between chromatin structure, cohesin activity and transcription
}

\author{
Douglas Maya-Miles ${ }^{1 \dagger}$, Eloísa Andújar ${ }^{2 \dagger}$, Mónica Pérez-Alegre ${ }^{2 \dagger}$, Marina Murillo-Pineda ${ }^{1,3}$, \\ Marta Barrientos-Moreno ${ }^{1}$, María J. Cabello-Lobato ${ }^{1,4}$, Elena Gómez-Marín' ${ }^{1}$ Macarena Morillo-Huesca' \\ and Félix Prado ${ }^{1 *}$ (i)
}

\begin{abstract}
Background: A complex interplay between chromatin and topological machineries is critical for genome architecture and function. However, little is known about these reciprocal interactions, even for cohesin, despite its multiple roles in DNA metabolism.

Results: We have used genome-wide analyses to address how cohesins and chromatin structure impact each other in yeast. Cohesin inactivation in scc 1-73 mutants during the $S$ and $G 2$ phases causes specific changes in chromatin structure that preferentially take place at promoters; these changes include a significant increase in the occupancy of the -1 and +1 nucleosomes. In addition, cohesins play a major role in transcription regulation that is associated with specific promoter chromatin architecture. In scc1-73 cells, downregulated genes are enriched in promoters with short or no nucleosome-free region (NFR) and a fragile "nucleosome -1/RSC complex" particle. These results, together with a preferential increase in the occupancy of nucleosome -1 of these genes, suggest that cohesins promote transcription activation by helping RSC to form the NFR. In sharp contrast, the scc1-73 upregulated genes are enriched in promoters with an "open" chromatin structure and are mostly at cohesin-enriched regions, suggesting that a local accumulation of cohesins might help to inhibit transcription. On the other hand, a dramatic loss of chromatin integrity by histone depletion during DNA replication has a moderate effect on the accumulation and distribution of cohesin peaks along the genome.
\end{abstract}

Conclusions: Our analyses of the interplay between chromatin integrity and cohesin activity suggest that cohesins play a major role in transcription regulation, which is associated with specific chromatin architecture and cohesinmediated nucleosome alterations of the regulated promoters. In contrast, chromatin integrity plays only a minor role in the binding and distribution of cohesins.

Keywords: Chromatin, Cohesin, Transcription, Scc1

\section{Background}

The first level of structural organization of chromosomes is the chromatin fiber, in which DNA is assembled into regularly spaced nucleosomes through the coordinated activity of histone chaperones, chromatin assembly factors and

*Correspondence: felix.prado@cabimer.es

${ }^{\dagger}$ Douglas Maya-Miles, Eloísa Andújar and Mónica Pérez-Alegre contributed equally to this work

${ }^{1}$ Department of Genome Biology, Andalusian Molecular Biology and Regenerative Medicine (CABIMER), CSIC-University of SevilleUniversity Pablo de Olavide, Seville, Spain

Full list of author information is available at the end of the article nucleosome modifiers and remodellers. Assembly of replicated DNA is coupled to the replication fork and occurs by the deposition of both newly synthesized and parental histones, which are distributed randomly between the sister chromatids [1]. In addition, replication-independent mechanisms of nucleosome assembly reset the chromatin changes induced by processes like transcription or DNA repair during the cell cycle $[2,3]$.

Chromatin fiber permits several levels of compaction of DNA into the nucleus, depending on the nuclear functional state or the cell cycle phase. Although the exact molecular structure behind compaction is still 
unclear, current models suggest that the packaging of chromatin requires dynamic and regulated interactions between irregularly folded fibers of nucleosomes, leading to a meshwork of intramolecular contacts [4]. The compaction, dynamics and regulation of this network rely mostly on the coordinated activities of topological machineries: cohesins, condensins and topoisomerases [5]. Whereas topoisomerases change the DNA supercoiling by cutting DNA molecules and re-sealing them in a new topological state [6], cohesins and condensins are ring-shaped molecules able to hold together distant chromatin fragments, thus compacting chromosomes [7].

In budding yeast, cohesins are loaded at chromatin by the cohesin loader complex Scc2/Scc4 during G1 and early $\mathrm{S}$ phase in a non-stable conformation that promotes topological and non-topological interactions (depending on whether or not cohesin entraps chromosomal DNA inside its ring) through dynamic turnover [8-10]. After loading, cohesins are moved away by the transcriptional machinery and accumulate preferentially at intergenic regions (IGRs) [11-14]. A fraction of cohesins becomes cohesive during $\mathrm{S}$ phase by topologically entrapping the two sister chromatids [15-18] and remains stably bound until its degradation in mitosis [19] (reviewed in [20, 21]). Although essential, sister chromatid cohesion is not the only function of cohesins, and multiple roles in DNA compaction, transcription regulation, DNA repair and DNA replication have been revealed in the past few years [22-25]. Remarkably, Scc2/Scc4 has been reported to have a role in RSC-mediated chromatin remodeling and transcription [26]. Specifically, the chromatin remodeling complex RSC recruits Scc2/Scc4 to specific promoters, where the cohesin loader helps to maintain the nucleosome-free region (NFR) for transcription activation [26]. RSC also interacts with and is required for cohesin loading [27] through a mechanism that involves direct interactions between RSC and both cohesin and Scc2/ Scc4 [28]. However, cohesins and cohesin loaders accumulate at non-overlapping peaks along the genome after their loading [12]. Therefore, it is unclear whether or not cohesins take part in chromatin remodeling and/or transcription in yeast.

Nucleosomes seem to have an inhibitory effect on cohesin binding. The Snf2-related complexes RSC and Irc5 in yeast, and SNF2h in humans are required for cohesin binding to chromosomes [27, 29, 30]. Importantly, although the chromatin remodeling activity of RSC is not necessary for cohesin loader recruitment, an ATPase dead RSC complex is defective for cohesin loading; accordingly, nucleosomes interfere with cohesin loading in vitro [28]. The distribution of cohesins is also associated with NFRs [31], although this preference might be an indirect consequence of their accumulation at IGRs [11, 13, 32], which contain NFRs [33, 34].

To address the connection between chromatin structure and cohesin function, we have now studied whether disrupting one affects the other. For this, we allow cells to progress from G1 to mitosis under conditions of histone depletion and/or lack of cohesin activity. Defective chromatin positioning by histone depletion had little or only region-specific effects on the accumulation and distribution of the major cohesin peaks. In contrast, the lack of cohesin activity affected the primary structure of chromatin at specific genomic regions, preferentially promoters. Critically, genome-wide analyses revealed a major role for cohesins in transcription regulation that is associated with the promoter chromatin architecture and location of the regulated genes.

\section{Results}

\section{Cohesins contribute to structuring chromatin}

To analyze what impact, if any, cohesins have on chromatin structure, we performed high-throughput sequencing of MNaseI-digested chromatin (MNase-seq) followed by dynamic analysis of nucleosome position and occupancy by sequencing (DANPOS) [35]. This approach allows nucleosomes to be mapped along the whole genome and categorizes the altered ones as changed in occupancy (measure of nucleosome density), position shift and fuzziness (degree of nucleosome deviation from its preferred position in a population). The experiment was performed with cells that express a thermosensitive allele of SCC1 (scc1-73). The $\alpha$-kleisin subunit Scc1 forms the tripartite ring-like structure with the SMC (structural maintenance of chromosomes) subunits Smc1 and Smc3 in the cohesin complex. The scc1-73 allele encodes a mutant protein $(\mathrm{S} 525 \mathrm{~N})$ that loses its ability to interact with $\mathrm{Smc} 1 / \mathrm{Smc} 3$ at $37^{\circ} \mathrm{C}$ and thereby causes cohesin inactivation $[8,36]$.

Cells were grown and synchronized in $\mathrm{G} 1$ at $26{ }^{\circ} \mathrm{C}$, released at $37^{\circ} \mathrm{C}$ and arrested in metaphase with nocodazole. This strategy ensures analysis of cells that have completed replication without cohesin activity and that have accumulated at the same cell cycle stage. The global profile of nucleosomes was similar in both $s c c 1-73$ and wild-type cells (Fig. 1a). Accordingly, the lack of cohesin activity did not affect the distribution of nucleosome fuzziness scores, neighboring distances or occupancy periodicities (Fig. 1b). However, some changes were observed when nucleosome occupancy was aligned for all genes relative to the transcription start site (TSS). Thus, although the pattern of nucleosome positioning at both sides of the promoter-associated NFR was apparently unaffected (Fig. 1c), the lack of cohesin activity caused a significant increase in the occupancy of nucleosomes -1 and +1 , but not in the occupancy of the nucleosomes in 


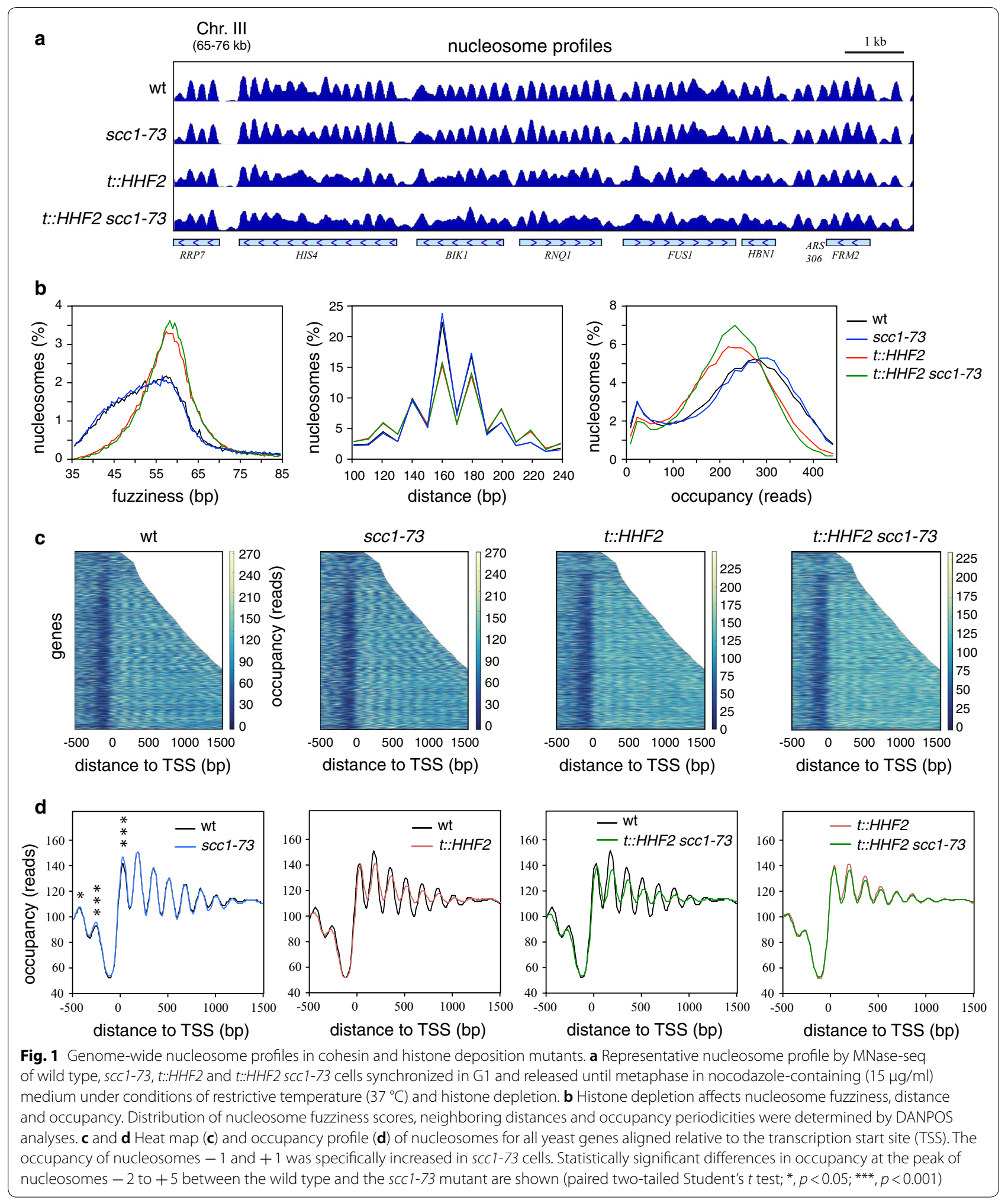

the gene body $(+2$ to +5$)$ (Fig. 1d, asterisks). In addition, DANPOS analysis revealed an elevated number of altered nucleosomes in scc1-73 cells as compared to wild-type cells $(\sim 1.1 \%$ of total nucleosomes; Table 1$)$. Most alterations affected the nucleosome occupancy, with a similar number of nucleosomes displaying either higher or lower 
Table 1 Number of altered nucleosomes (in position, fuzziness and/or occupancy) upon histone depletion (in t::HHF2 cells) or cohesin inactivation (in scc1-73 cells) as compared to the profile of nucleosomes in wild-type cells

\begin{tabular}{lccc}
\hline & t::HHF2 & scc1-73 & t::HHF2 scc1-73 \\
\hline Altered nucleosomes & $4431^{*}$ & 768 & $6151^{*}$ \\
Position shift & 319 & 35 & 389 \\
Fuzziness change & 1516 & 77 & 2093 \\
Occupancy change & 3989 & 703 & 5618 \\
Occupancy increase & 1304 & 375 & 2080 \\
Occupancy decrease & 2685 & 328 & 3538
\end{tabular}

* The percentage of altered nucleosomes between $t:: H H F 2$ cells (6.68\%) and scc1-73 t::HHF2 cells (9.28\%) was statistically different according to a two-tailed Chi-square test $(p<0.001)$

occupancy than in wild-type cells. Further analysis of the altered nucleosome distribution in the genome showed that they were enriched in IGRs, tRNA genes and telomeres; averagely distributed in the pericentric chromatin and rDNA; and had a less than average distribution in the ORFs (Additional file 1: Table S1 and Additional file 2: Table S2). We conclude that cohesins contribute to establish the primary structure of chromatin at specific DNA regions, preferentially promoters.

Lack of cohesin activity has a major effect in transcription that is linked to specific chromatin structure at promoters As the effect of $s c c 1-73$ was more prominent on the chromatin of IGRs and the occupancy of nucleosomes -1 and +1 , we asked if those chromatin changes are associated with alterations in the pattern of transcription regulation. We synchronized wild-type and scc1-73 cells in $\mathrm{G} 1$ and then released them into fresh medium until metaphase under restrictive conditions, to compare their genome-wide transcription profiles by RNA-seq under the same conditions that were used to study their chromatin structure. The absence of cohesin activity from G1 to metaphase in the $s c c 1-73$ mutant yielded an elevated number of misregulated genes, with 445 downregulated genes and 569 upregulated genes ( $q$ value $<0.01$; 1.41 fold cutoff relative to wild type); this represents $\sim 15 \%$ of all yeast genes. (Noncoding RNAs were not affected.) Of these, 255 genes ( 4\%; 91 downregulated and 164 upregulated) showed a $>2$-fold change (Fig. 2a and Additional file 3: Table S3). These genes showed a significant overrepresentation of gene ontology biological process terms related to RNA and amino acids metabolism and stress responses (Additional file 3: Table S3).

Next, we asked whether gene regulation by cohesins is associated with chromatin alterations. Misregulated genes upon cohesin inactivation in the $\operatorname{scc} 1-73$ mutant were not associated with DANPOS-defined altered nucleosomes, indicating that these alterations were not caused by defective transcription. The only exception was a slight enrichment in nucleosomes with higher occupancy at the promoters of the downregulated genes (Fig. 2b). In line with this, the increase in the occupancy of nucleosome -1 remained significant in the $s c c 1-73$ downregulated (but not upregulated) genes, even though it was lost in control groups (chromosomes VII and XII) with a similar number of genes (Fig. 2c). (Note that the number of genes analyzed in Fig. 1d was $\sim 10$ times higher than that in Fig. 2c.) The downregulated genes in $s c c 1-73$ cells were also characterized by significant changes at the nucleosomes downstream of the promoter, which might result from defective transcription. In contrast to these changes, the increase in the occupancy of nucleosome +1 was also detected in control groups of transcriptionally unaffected genes (Fig. 2c, chromosomes VII and XII), indicating that it was transcription independent.

We next addressed whether the effects that a lack of cohesin activity had on transcription were associated with the promoter chromatin architecture. In yeast, gene promoters can be grouped according to their chromatin structure. One of these classifications distinguishes genes with occupied-proximal nucleosome (OPN) and depleted-proximal nucleosome (DPN) promoters, depending on the absence or presence of an NFR just upstream of nucleosome +1 [37]. A similar analysis defines a higher number of genes as "open" or "closed" depending on the presence or absence of a clear NFR at the promoter [38]. Notably, scc1-73 downregulated genes were highly enriched in OPN and "closed" genes (Table 2).

More recent and exhaustive studies involving deep sequencing of MNaseI-treated DNA at different degrees of digestion uncovered two groups of promoters, defined according to the stability of nucleosome -1 : $(i)$ promoters with a nuclease-resistant nucleosome (stable nucleosome, SN), which have a constitutive NFR of less than $150 \mathrm{bp}$, and (ii) promoters with a nuclease-sensitive nucleosome (fragile nucleosome, FN), which is removed through the action of transcription factors; these factors, together with the chromatin remodeling complex RSC, lead to an NFR of more than 150 bp upon transcription activation $[39,40]$. Notably, scc1-73 downregulated genes are highly enriched in FN promoters (Table 2) and accordingly in promoters with RSC/nucleosome complexes [41] (Table 2). Finally, we asked if scc1-73 downregulated genes were preferentially associated with Scc2/Scc4-regulated genes, as RSC-mediated chromatin remodeling and transcription activation requires $\mathrm{Scc} 2 /$ Scc4 promoter binding [26]. We did not find a preferential association between Scc2-binding genes and 


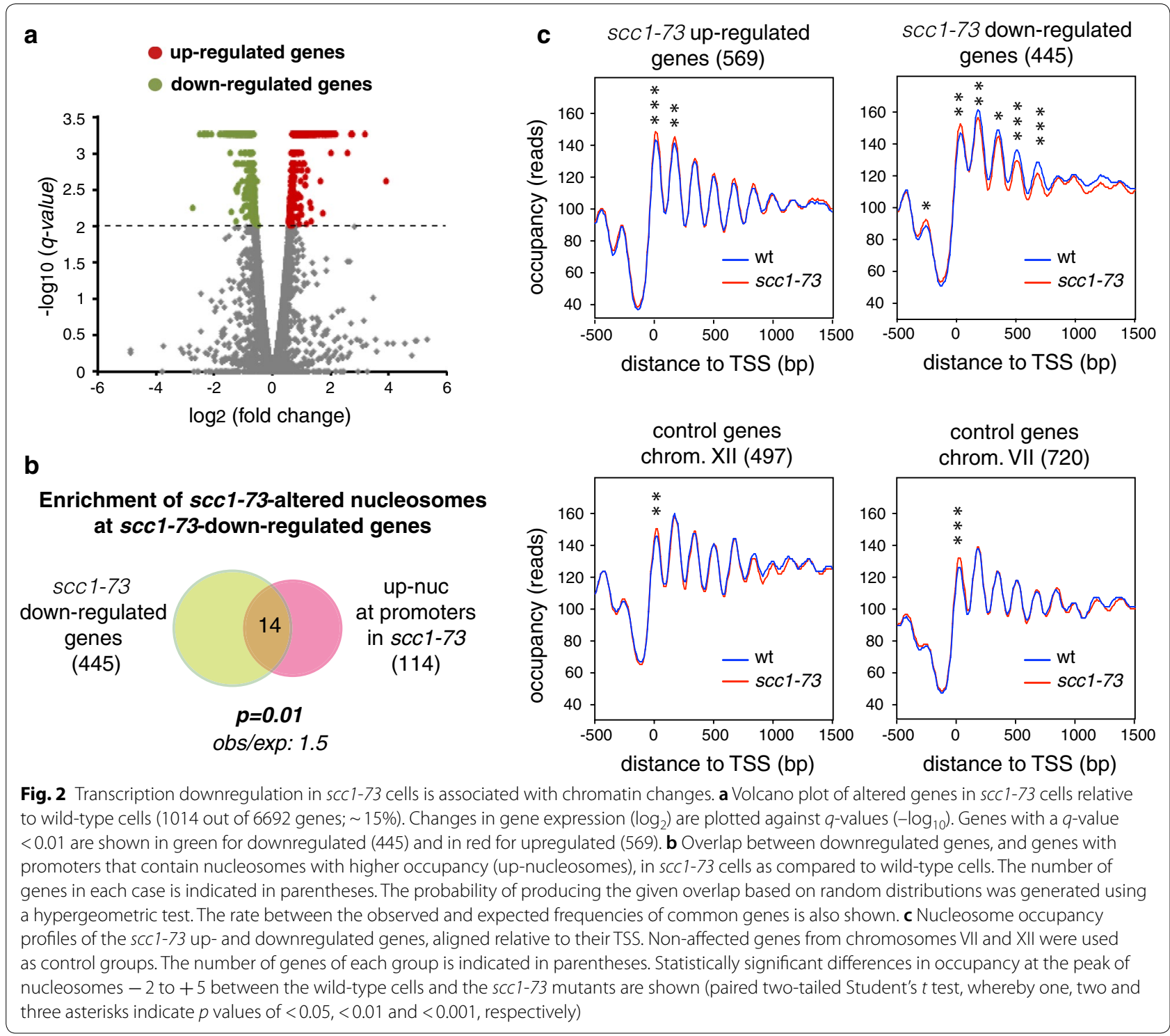

scc1-73-misregulated genes (Table 2). Therefore, transcription activation by cohesins seems to be linked to specific chromatin structure at promoters. These results, together with a preferential increase in the occupancy of nucleosome -1 of scc1-73-downregulated genes (Fig. 2c), suggest that cohesins promote transcription activation by helping RSC to form the NFR.

Approximately $20 \%$ of yeast genes contain a TATA box [42]. This element is enriched in the OPN genes and underrepresented in the DPN genes [37], and accordingly, the TATA box was highly enriched among the downregulated genes in scc1-73 cells (Table 2). Early studies analyzing steady-state RNA levels showed that TATA-containing genes preferentially use the SAGA complex rather than the general transcription factor
TFIID [42]. Similar studies have revealed a predominant role for TFIID ( 90\% of genes) and a more restricted role for SAGA ( $10 \%$ of genes) [43]. More recent analyses have also shown that the Mediator complex is particularly important for the expression of TATA-containing, SAGA-dominated genes, whereas the histone acetyltransferase complex NuA4 is preferentially associated with TFIID [44, 45]. As expected from these associations, the set of downregulated genes in scc1-73 cells were dominated by the Mediator and SAGA and not by NuA4 and TFIID; in addition, they were enriched in SWR1dominated genes [46] (Table 2). In marked contrast with scc1-73-downregulated genes, scc1-73-upregulated genes were enriched in genes with "open" promoters and dominated by TFIID and NuA4 (Table 2). Even though 


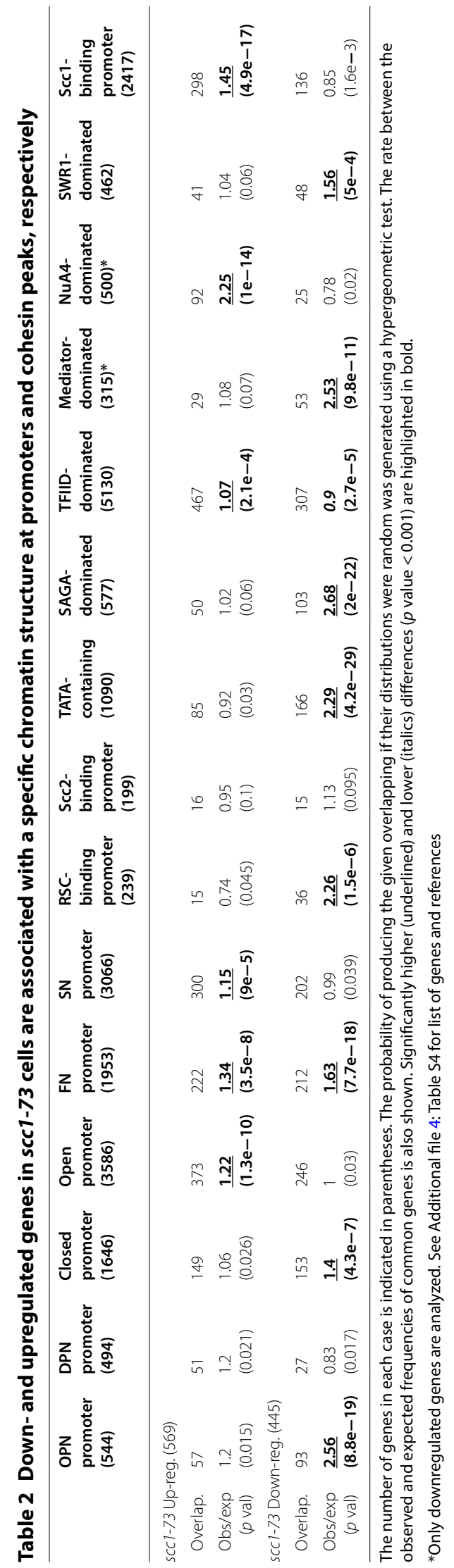


these associations may reflect different mechanisms of gene regulation, they connect the role of cohesin in gene expression with specific configurations of transcriptional regulators at promoters.

\section{The lack of cohesin activity and histone depletion leads to similar occupancy changes in a subset of nucleosomes} Our group has previously reported that the increased accessibility to MNaseI of the centromeric chromatin at CEN3 that can be observed in histone-depleted cells is partially suppressed by cohesin inactivation, suggesting that cohesins could contribute to the loss of chromatin integrity associated with defective histone deposition [47]. To further understand the function of cohesins in chromatin organization, we analyzed the genome-wide effects of the scc1-73 allele in cells that express histone $\mathrm{H} 4$ from the doxycycline (dox)-regulatable tet promoter $(t:: H H F 2)$ [48]. For this, $t:: H H F 2$ and $t:: H H F 2$ scc1-73 cells were released together with $s c c 1-73$ and wild-type cells from G1 under conditions of histone depletion and restrictive temperature (Fig. 1). Cells depleted of histone H4 during $\mathrm{S}$ phase displayed a severely altered nucleosome profile in metaphase (Fig. 1a), which was especially enriched in nucleosomes with reduced occupancy (Table 1). Histone depletion caused a global increase in fuzziness and a wider distribution of both nucleosome occupancy and distance between adjacent nucleosomes (Fig. 1b). The overall loss of nucleosome positioning became particularly evident when nucleosome occupancy was aligned for all genes relative to the TSS, where the precise pattern of nucleosome positioning at both sides of the promoter-associated NFR was lost following histone depletion (Figs. 1c, d). These results are in accordance with a previous study showing that defective histone supply after H3 depletion strongly affects chromatin integrity genome-wide [49].

The double-mutant $t:: H H F 2$ scc1-73 displayed a large number of chromatin perturbations (Fig. 1a, d). This loss of chromatin integrity at $t:: H H F 2$ cells was confirmed at three different regions by indirect end labeling of MNaseI-treated cells (Additional file 8: Fig. S1). Importantly, the lack of Scc1 activity increased the number of genome-wide altered nucleosomes in histone-depleted cells (Table 1; compare $t:: H H F 2$ scc1-73 with $t:: H H F 2$ ), consistent with an additive effect of the lack of histones and cohesin activity. Thus, the partial suppression of nucleosome alterations at CEN3 described previously was specific for that locus [47]. We could not check these changes in our MNase-seq experiments because the centromeric nucleosomes were not detected (as previously reported in similar studies $[49,50])$.

A comparative analysis showed that both the profile of genomic regions with altered nucleosomes and the class of changes in nucleosome occupancy in these genomic regions coincided in many cases in scc1-73 and $t:: H H F 2$ (Additional file 1: Table S1 and Additional file 2: Table S2). This observation prompted us to study if nucleosomes with altered occupancy lie at the same genomic regions in both mutants. Statistical analyses of IGRs with altered nucleosomes showed a highly significant number of common elements (Fig. 3a). Importantly, overlapping IGR shared nucleosomes with the same change-either increased or decreased-in occupancy (Fig. 3b). Similar results were obtained from the analysis of ORF with altered nucleosomes (Fig. 3c, d). A deeper analysis showed that this overlap was due to the fact that $t:: H H F 2$ and $s c c 1-73$ share a highly significant number of altered nucleosomes with the same change (Fig. 3e, f), especially in nucleosomes with increased occupancy (8 to 10 times more than expected). Moreover, manual inspection of these nucleosomes showed that the change in the double-mutant $t:: H H F 2 s c c 1-73$ was similar to that displayed by the single mutants for $92 \%$ of nucleosomes (see representative examples, Fig. 3e, f). Altogether, these results suggest that part of the chromatin changes induced by defective nucleosome assembly and cohesin inhibition occurs through the same mechanism ( 17\% of scc1-73-altered nucleosomes).

\section{Mutant scc 1-73 cells do not display defects in the deposition of newly synthesized histones during DNA replication}

The epistatic effect of $s c c 1-73$ and $t:: H H F 2$ on the integrity of a subset of nucleosomes might reflect a role for cohesins in histone deposition. Indeed, our RNA-seq analysis showed that the scc1-73 mutant was specifically affected for expression of histones $\mathrm{H} 4$ and Htz1, as well as of histone chaperones Nap1 and Chz1 (Additional

\footnotetext{
(See figure on next page.)

Fig. 3 A lack of cohesin activity and histone depletion lead to similar occupancy changes in a subset of nucleosomes. a, c Overlap between IGR (a) or ORF (c) with altered nucleosomes in t::HHF2 or scc1-73 cells. b, d Overlap between IGR (b) or ORF (d) with either up- (increased occupancy) or down-nucleosomes (decreased occupancy) in t::HHF2 and scc1-73. e, f Overlap between up- (increased occupancy) or down-nucleosomes (decreased occupancy) in IGR (e) and ORF (f) in t::HHF2 and scc1-73 cells. Representative examples are shown; up- and down-nucleosomes are marked in green and red, respectively. The number of genomic regions $(\mathbf{a}-\mathbf{d})$ or nucleosomes $(\mathbf{e}, \mathbf{f})$ in each case is indicated in parentheses. The probability of producing the given overlap if their distributions were random was generated using a hypergeometric test. The rate between the observed and expected frequencies is also shown
} 


\section{a}

IGR with altered nucleosomes in both $t:: H H F 2$ and scc1-73

$$
\begin{gathered}
\text { IGR with } \\
\text { altered nuc } \\
\text { in } t:: H H F 2(1180)
\end{gathered}
$$

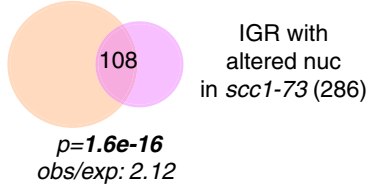

b

IGR with similar and opposite nucleosome occupancy changes in $t:: H H F 2$ and scc1-73

\section{IGR with up-nuc}

in $t:: H H F 2(504)$

$$
\begin{gathered}
p=0.03 \\
\text { obs/exp: } 1.49
\end{gathered}
$$

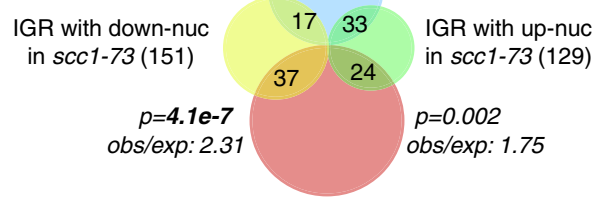

IGR with down-nuc

in $t:: H H F 2(700)$

e

Common altered nucleosomes in $t:: H H F 2$ and scc1-73 (at IGR)
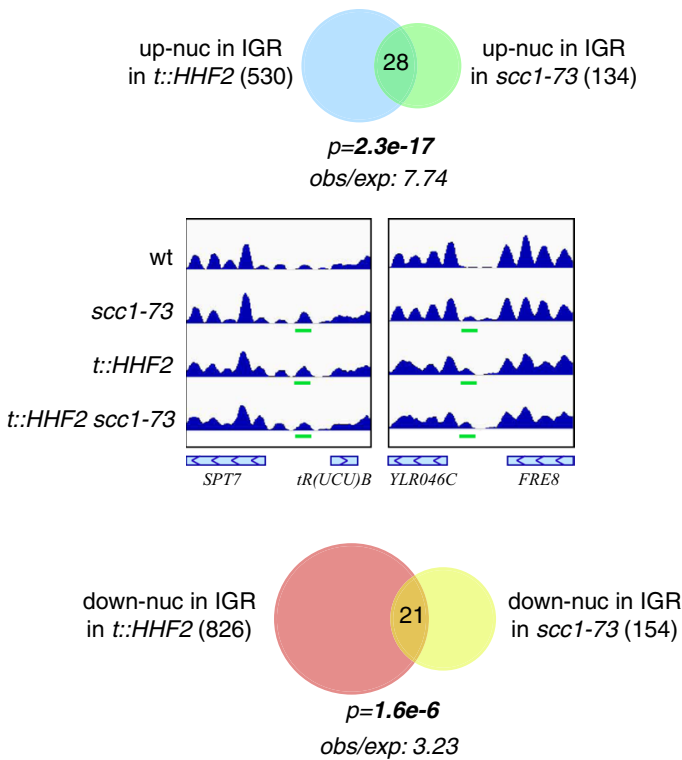

obs/exp: 3.23

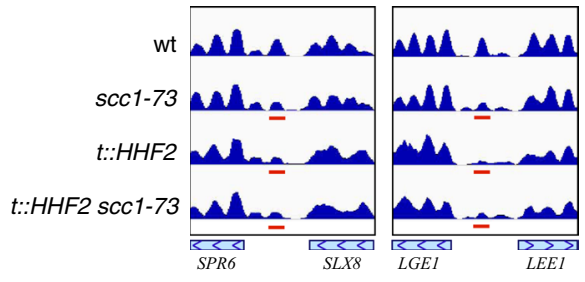

c

ORF with altered nucleosomes in both $t:: H H F 2$ and scc1-73

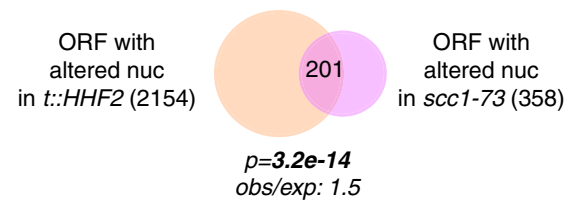

d

ORF with similar and opposite nucleosome occupancy changes in t::HHF2 and scc1-73

\section{ORF with up-nuc}

in $t:: H H F 2(647)$

$$
\begin{array}{cl}
p=0.03 & p=1.5 e-21 \\
\text { obs/exp: } 1.29 & \text { obs/exp: } 3.57
\end{array}
$$

ORF with down-nuc $\quad 30 \quad 63 \quad$ ORF with up-nuc

in $\operatorname{scc} 1-73(206) \quad 80 \quad 48$ in $\operatorname{scc} 1-73(157)$

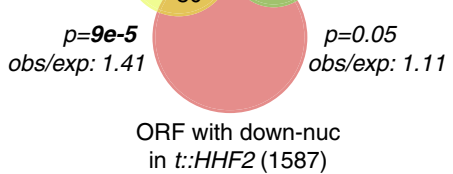

Common altered nucleosomes in $t:: H H F 2$ and scc1-73 (at ORF)
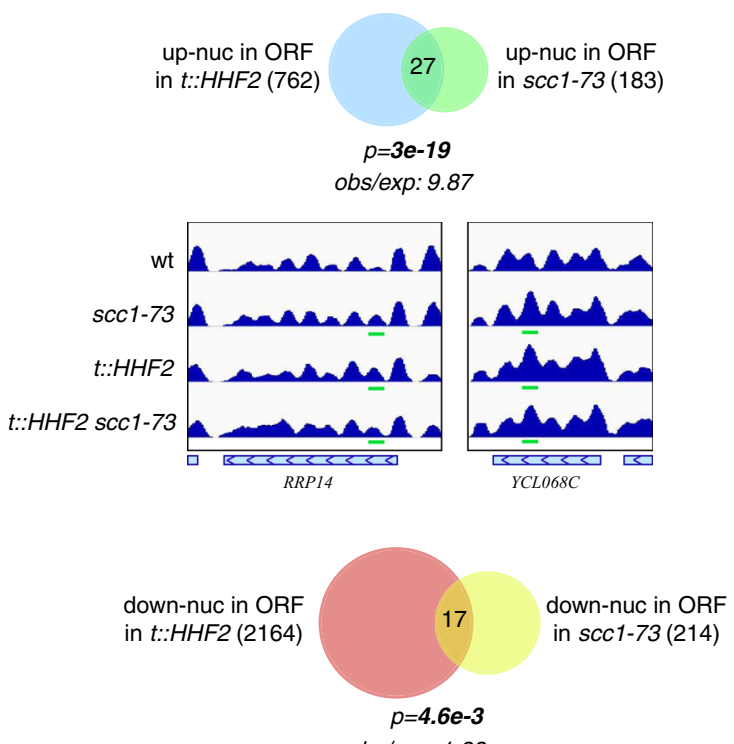

obs/exp: 1.88

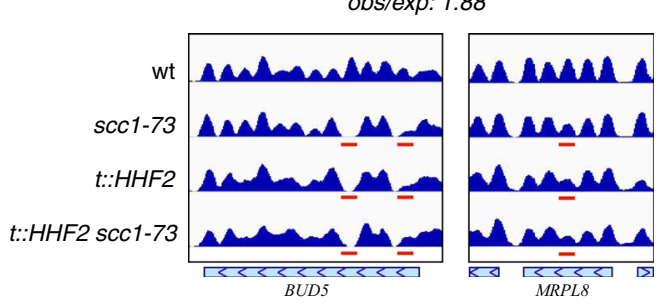


file 3: Table S3). To determine whether cohesins regulate histone deposition, we analyzed the incorporation into chromatin of newly synthesized histones at replicating DNA regions by following acetylated $\mathrm{H} 3$ at lysine K56 (H3K56ac) [51]. Although only a few nucleosomes were affected in $s c c 1-73$ cells, we speculated that recycling parental histones could mask a major defect in the deposition of newly synthesized histones.

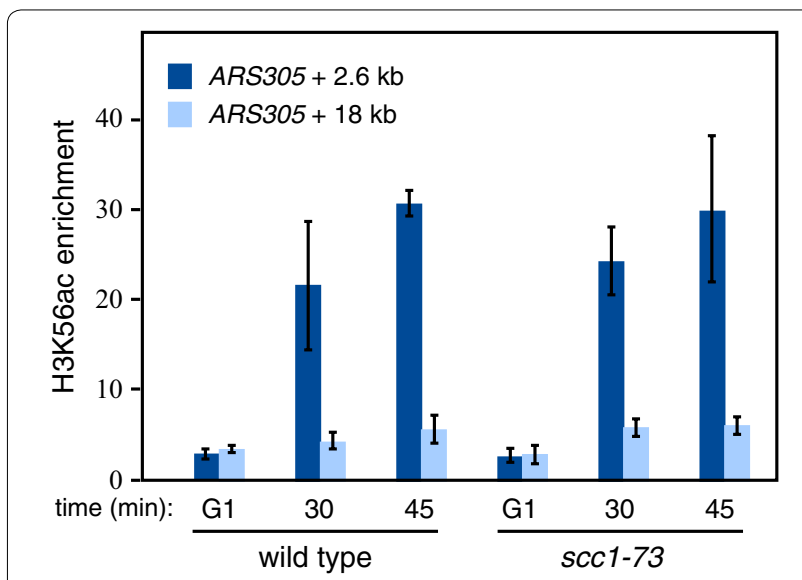

Fig. 4 Cohesins are not involved in the deposition of newly synthesized histones. ChIP analysis of the incorporation of newly synthesized histone $\mathrm{H} 3$ (acetylated in lysine 56) in wild-type and scc1-73 cells that have been synchronized in $\mathrm{G} 1$ and released into $S$ phase in the presence of $200 \mathrm{mM} \mathrm{HU}$ for $45 \mathrm{~min}$. H3K56c enrichment both at the proximity of ARS305 and at an unreplicated region (2.6 and $18 \mathrm{~kb}$ from the origin, respectively) was calculated as the amount of DNA immunoprecipitated with an antibody against $\mathrm{H3K} 56 \mathrm{aC}$ relative to that obtained with antibody against total histone $\mathrm{H} 3$. The average and range from two independent experiments are shown. lgG-treated cells were used as an internal control to confirm the specific enrichment at each region
H3K56ac accumulation at replicating DNA regions from origin ARS305 was analyzed in cells synchronized in G1 and released into $S$ phase in the presence of hydroxyurea, which reduces the pool of dNTPs and causes replication forks to be stalled in the proximity of the origin [52]. As expected, H3K56Ac accumulated around the origin (2.6 kb from ARS305) but not at an unreplicated DNA region (18 Kb from ARS305). Importantly, the absence of cohesin activity in scc1-73 cells did not affect H3K56ac incorporation (Fig. 4), suggesting that cohesins do not have a major role in depositing new histones during DNA replication.

\section{Histone depletion has modest effects on the accumulation and distribution of cohesin peaks}

To further understand the interplay between cohesins and chromatin structure we studied the importance of chromatin integrity in cohesin binding and distribution. For this, cells were synchronized in G1 and released into fresh medium until G2/M under conditions of histone depletion at $30{ }^{\circ} \mathrm{C}$, and the association of Scc1 was followed by a high-density ChIP-on-chip analysis (5-bp resolution). This analysis showed an overall similarity of cohesin peaks in t::HHF2 and wild-type cells (Fig. 5a, b and Additional file 9: Fig. S2a), with cohesins preferentially bound to the pericentromeric chromatin, rDNA, tRNA genes and the IGR of convergently transcribed genes (Fig. 5a-c) [11-13]. However, the amount of cohesins at rDNA, tRNA genes and telomeres relative to the genome average was higher in histone-depleted cells than in wild-type cells (Fig. 5d and Additional file 5: Table S5). As the ChIP-on-chip analysis was not normalized with an internal control, no comparison between absolute amounts of signal between wild-type and mutant could

(See figure on next page.)

Fig. 5 Histone depletion has a moderate effect on cohesin binding and distribution. a Cohesin distribution at chromosome I in wild-type and t::HHF2 cells synchronized in G1 and released into fresh medium until G2-metaphase, as determined by ChIP-on-chip analysis against Scc1-HA. b Cohesin distribution at the ribosomal DNA locus in wild-type and t:.:HHF2 cells. c Number of IGRs classified according to the orientation of the flanking genes that overlap with cohesins (by at least $1 \mathrm{bp}$ ) in wild-type and $t:: H H F 2$ cells. $\mathbf{d}$ Relative amount of cohesins in $t:: H H F 2$ cells relative to wild-type cells at the indicated genomic regions. The total amount of cohesins at each region was calculated considering the sum of positive signals (relative to the untagged strain) with a $p<0.05$. The ratio between the mutant and the wild-type at each region was normalized to that obtained for the whole genome, which was taken as 1. The proportion of cohesins between mutant and wild-type cells at each genomic region relative to the genome average was statistically different according to a two-tailed Chi-square test $(p<0.001)$. e Cohesin enrichment in $t: .: H H F 2$ cells relative to wild-type cells at different genomic regions, as determined by ChIP and qPCR analyses against HA-Scc1 in cells grown as in $\mathbf{a}$. IGR and tRNA genes are indicated in Methods section. Cohesin enrichment was calculated as the ratio between immunoprecipitated DNA and input in t::HHF2 cells relative to the same value in the wild-type cells. The average and SEM from 3 to 4 independent experiments are shown. An untagged strain was used as an internal control to confirm the specific enrichment at each region. The amount of Scc1 relative to Pgk1 is shown on the left. No significant differences were observed between wild-type and mutant cells from four independent measurements. $\mathbf{f}$ Number of IGRs, ORF, tRNA, ARS and telomeres that overlap with cohesins (by at least 1 bp) in wild-type and t::HHF2 cells. $\mathbf{g}$ Probability that scc 1-73 alters cohesin-associated nucleosomes if scc1-73-altered nucleosomes were randomly distributed, as determined by a hypergeometric test. The rate between the observed and expected frequencies of common nucleosomes is also shown. The number of nucleosomes in each case is indicated in parentheses. $\mathbf{h}$ Comparison of the percentages of altered nucleosomes by histone depletion (showing occupancy, fuzziness and position shift) in the regions with cohesins (9596 nucleosomes) relative to the whole genome (66,278 nucleosomes) in t::HHF2 cells. A hypergeometric test was used to determine the probability of obtaining the indicated percentages of altered nucleosomes at regions with cohesin if their distributions were random 


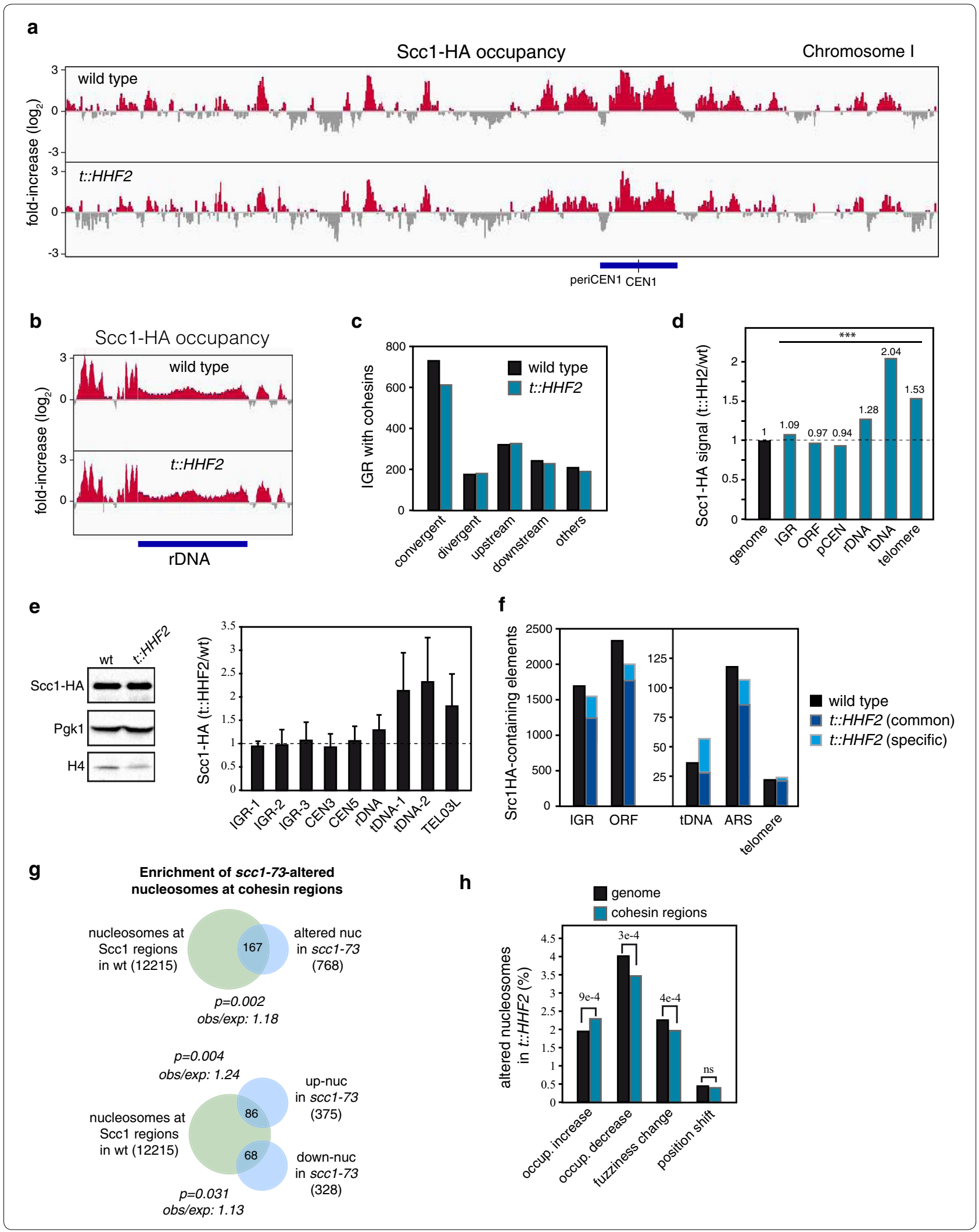


be made; thus, we performed quantitative PCR from ChIP samples to validate the changes observed in cohesin binding. The absolute amount of cohesins at the major sites of cohesin binding (IGR and centromeres) was not affected, whereas higher levels of cohesins were detected at rDNA, tRNA genes and telomeres (Fig. 5e). While telomeres and rDNA were enriched in nucleosomes with increased and decreased occupancy, respectively, the percentage of altered nucleosomes at tDNA genes was below the whole genome average (Additional file 1: Table S1 and Additional file 2: Table S2).

Apart from these region-specific changes, the genomewide analysis showed a loss of cohesins in 465 IGRs, and a gain of cohesin peaks in 315 IGRs that did not accumulate cohesins in the wild-type cells. Similar redistributions of cohesins were observed in ORF, tDNA genes, ARS and telomeres (Fig. 5f). This redistribution was not associated with preferential alterations in chromatin relative to non-affected regions, except for a slight increase in nucleosomes with higher occupancy (Additional file 9: Fig. S2b). Likewise, there was no significant overlap between IGR that had lost cohesins in $t:: H H F 2$ and the IGR with altered nucleosomes in both $t:: H H F 2$ and $s c c 1$ 73 (Additional file 9: Fig. S2b), suggesting that shared nucleosome alterations are not due to the loss of cohesin peaks in histone-depleted cells. In sum, defective chromatin integrity in histone-depleted cells has a moderate impact on cohesin binding, which is mostly evidenced by a redistribution of a subset of cohesin peaks and a higher accumulation at specific genomic regions.

\section{Cohesins have a slight contribution to maintaining chromatin integrity at cohesin-enriched regions}

The MNase-seq analysis showed that cohesins contribute to the formation of a correct chromatin structure at specific genomic regions. To gain insight into the effects of cohesins on chromatin structure, we analyzed the position of altered nucleosomes in scc1-73 cells relative to the distribution of cohesins in wild-type cells. We found that nucleosomes that overlap with cohesin regions have a slight but significant higher probability of being altered in scc1-73 cells (Fig. 5g). We observed the same effect after analyzing genomic regions with altered nucleosomes in scc1-73 cells; namely, altered nucleosomes were preferentially at IGR and ORF that contained cohesin peaks in wild-type cells (Additional file 6: Table S6). Thus, the loss of chromatin integrity upon cohesin inactivation is slightly more severe in cohesin-enriched regions.

Finally, a cohesin distribution analysis allowed us to address how cohesins influence chromatin disruption by histone depletion. A comparative analysis with the whole genome showed that regions with cohesins display higher levels of nucleosomes with increased occupancy and lower levels of nucleosomes with decreased occupancy and increased fuzziness (Fig. 5h), suggesting that cohesins prevent the loss of nucleosome positioning upon histone depletion.

\section{Upregulated genes in scc1-73 cells are enriched in cohesins} As the elimination of cohesin activity during $S$ phase affects gene expression, we asked whether this misregulation was associated with a direct binding of cohesins to regulated genes. Remarkably, upregulated, but not downregulated, genes in scc1-73 cells displayed a highly significant enrichment in cohesin-binding genes (Table 2), suggesting that cohesin peaks have a repressor role.

\section{Discussion}

\section{Cohesins contribute to structuring chromatin}

Here we provide evidence that cohesins help to determine the primary chromatin structure of specific DNA regions. The absence of cohesin activity in the $s c c 1-73$ mutant altered the occupancy of hundreds of nucleosomes. This effect was more pronounced in IGR, leading to both gain and loss of nucleosome occupancy. In addition, the occupancy of nucleosomes -1 and +1 at promoters was specifically increased on average in a transcription-independent $(+1)$ and partially dependent $(-1)$ manner. These results suggest that cohesins have a subtle but significant effect on the architecture of yeast promoters. Formally, we cannot rule out that some of the chromatin changes in the $s c c 1-73$ mutant stem from transcriptional defects, although the use of a conditional mutant reduces this possibility. Note that this does not mean that cohesins affect nucleosome positioning and/ or transcription by acting directly on the altered nucleosomes and/or genes; our results show that a lack of cohesin activity affects the integrity of nucleosomes at both cohesin-associated and cohesin-free regions. Cohesins might help to shape cohesin-associated chromatin regions by either preventing histone deposition and/or facilitating nucleosome positioning, depending on additional chromatin determinants. Likewise, the effects of cohesin on nucleosomes at "cohesin-free" regions (as defined here and in other studies [11-13]) might be direct, as analyzing these regions with more sensitive methods reveals an accumulation of basal levels of cohesins [53]. Alternatively, nucleosome alterations at cohesin-free regions might result from cohesin activity at a distance, through topological constrains on adjacent chromatin fragments [54], as cohesins are major determinants of chromatin cis-loops from human to yeast [55-57]. Finally, chromatin defects might be indirectly generated by impairment of other cohesin-regulated processes. Similar arguments are valid for the effects of scc1-73 in transcription. 
Notably, a significant number of altered nucleosomes in scc1-73 cells $(\sim 17 \%)$ were shared by $t:: H H F 2$ and $t:: H H F 2$ scc1-73 cells, suggesting that they occur through a common mechanism. We have discarded a major role for cohesins in the deposition of newly synthesized histones, even though it is still possible that the absence of cohesins affects recycling of parental histones, as sister chromatid entrapment by cohesin during replication and parental histone deposition are both associated with the replication fork [1, 58-61].

\section{Transcription regulation by cohesins}

Studies in Drosophila and vertebrates using cohesin mutants have revealed both up- and downregulation of genes controlling development, proliferation and pluripotency [23, 62]. Our genome-wide transcription analysis showed a high number of misregulated genes in the scc1-73 mutant $(\sim 15 \%$ with a $>1.5$-fold change; $\sim 4 \%$ with a $>2$-fold change); these numbers are in the range of those obtained by the absence of the chromatin remodelers SWR or RSC, which have genome-wide transcriptional roles (7\% and $17 \%$ with $>1.5$-fold change for swr $1 \Delta$ and sth1-3, respectively) $[26,46]$. Indeed, a global gene expression analysis of 132 mutants of chromatin regulators with altered transcription profiles showed that the average percentage of genes with a significant change (fold change $>1.7$ ) was $\sim 2 \%$ and that the largest effect was $16 \%$ [63]. Therefore, our results support a major role for cohesins in transcription regulation in yeast.

We do not rule out that cohesins play a role in transcription by promoting DNA looping, as proposed for vertebrates [64]. However, transcription analyses in the scc1-73 mutant relative to cohesin accumulation and chromatin structure, together with the effects of cohesins on nucleosome occupancy at promoters, suggest additional (but not mutually exclusive) mechanisms of transcription regulation by cohesins. First (and in sharp contrast to $s c c 1-73$ downregulated genes, which show a random distribution), upregulated genes in $s c c 1-73$ cells mainly are in cohesin-enriched regions. The nucleosome profile of upregulated genes in scc1-73 cells was not associated with specific chromatin changes. One possibility is that cohesins facilitate transcription repression by compacting chromatin without altering nucleosome occupancy. Alternatively, cohesin accumulation might inhibit transcription by specifically recruiting repression factors and/or hampering the binding and/or movement of the transcription machinery (Fig. 6a). In any case, it is particularly interesting that upregulated genes in $s c c 1-73$ cells are enriched in genes that require the NuA4 complex for activation, as it opens the possibility that histone acetylation is required to counteract a putative repressor role by cohesins.

On the other hand, downregulated genes in scc1-73 cells were characterized by promoters containing TATA and regulated by the Mediator, SAGA and SWR complexes. This suggests that, regardless of the putative mechanisms of action, chromatin dynamics plays a major function in the activation of these genes. Critically, the role of cohesins in transcription activation seems to be associated with a specific chromatin structure at promoters. Thus, downregulated genes were enriched in promoters with short or no NFR and a fragile nucleosome at the position of nucleosome -1 . The proposal of this fragile nucleosome [39] has been challenged by data supporting the presence of non-histone protein complexes [65]. However, recent analysis revealed the presence of the RSC complex and global transcription factors bound to a partially unwrapped nucleosome intermediate [66]. In addition to removing this nucleosome, these factors

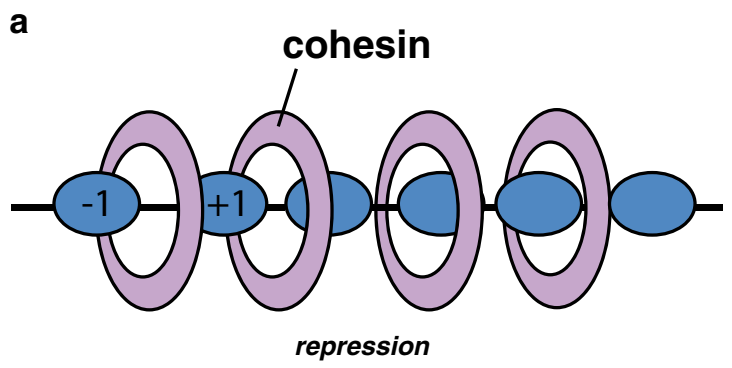

b

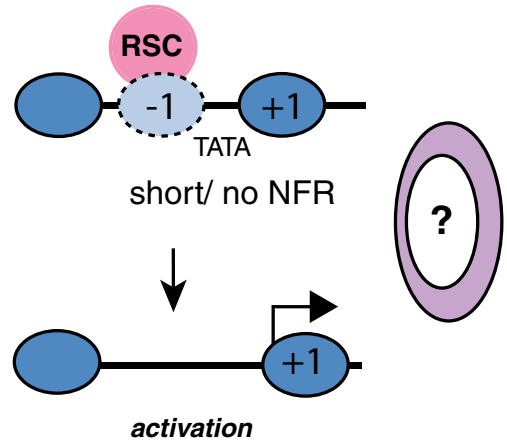

Fig. 6 Hypothetical model for gene regulation by cohesins. a Upregulated genes in scc1-73 cells are preferentially in cohesin-enriched regions. Cohesin accumulation might recruit transcription repressors, generate a locally condensed chromatin or hamper the recruitment and/or movement of the transcription machinery. $\mathbf{b}$ Downregulated genes in scc 1-73 cells are enriched in promoters that have a short or no NFR and a fragile -1 nucleosome. These promoters require the RSC complex together with additional transcriptional factors to generate a NFR and to activate transcription. Cohesins might collaborate with RSC and other chromatin remodeling factors to remove the nucleosome and/or maintain the NFR 
seem to shift apart the flanking nucleosomes, exposing the TBP-binding site, which facilitates transcription (Fig. 6b) [39, 40, 66, 67]. In accordance with a putative role for cohesins in the regulation of genes with FN promoters, downregulated genes in scc1-73 cells were enriched in RSC/nucleosome complexes at their promoters. Interestingly, the occupancy of nucleosome -1 was particularly increased in scc1-73-downregulated genes. Taking into account these associations, one possibility is that cohesins help RSC to remove the nucleosome -1 and/or to maintain the NFR (Fig. 6b). In this light, it is worth noting that RSC facilitates the recruitment to promoters of the cohesin loader Scc2/Scc4, which in turn helps RSC to maintain the NFR [26]. Moreover, RSC interacts directly with both cohesin and Scc2/Scc4 and is required for cohesin loading $[27,28]$. However, the Scc2/Scc4 complex was not preferentially associated with downregulated genes in scc1-73 cells, suggesting that cohesins operate in transcription independently of Scc2/ Scc4. Alternatively, transient and dynamics interactions between RSC, Scc2/Scc4 and cohesins may be required for chromatin remodeling and transcription regulation. Further genomic and gene-specific time-course molecular analyses will be required to validate these hypothetical models, and to determine if the role of cohesins in transcription in S. cerevisiae is also related to its DNA looping activity.

The increase in occupancy at nucleosome -1 is more severe (but not specific) for scc1-73-downregulated genes. Further, the lack of cohesin activity causes a global effect on the occupancy of nucleosome +1 , a nucleosome that plays a key role in the regulation of transcription. Nascent mRNA studies will be required to establish if the effects of $s c c 1-73$ on nucleosomes -1 and +1 are associated with a more general role of cohesins in transcription regulation.

\section{Chromatin integrity plays a moderate role in global cohesin binding and distribution}

Until now, whether (and how) chromatin structure contributes to cohesin binding and distribution has been controversial. Recently, Uhlmann and colleagues provided strong in vivo and in vitro evidence for an inhibitory role of nucleosomes in cohesin loading [28]. Here, we show that the amount of cohesins was not increased in most genomic regions after histone depletion and that there was not a preferential loss of nucleosome occupancy in those regions in which cohesins accumulated relative to the wild type. Therefore, the amount of loaded cohesins is not limited by chromatin under wild-type conditions. Remarkably, the general pattern of cohesin peaks as well as the preferential regions of cohesin binding remained mostly unaffected despite a severe loss of chromatin integrity. Therefore, the global pattern of cohesins throughout the genome does not seem to require a precise chromatin organization. Furthermore, the number of cohesin peaks did not augment despite a global reduction in nucleosome density, suggesting that NFRs per se are not preferential sites for cohesin accumulation. Although these results do not discard the requirement of specific histone interactions for cohesin binding and/or distribution, they point to DNA-associated specific features (e.g., A/T-rich DNA sequences, positively supercoiled DNA) $[14,68]$ and DNA metabolic processes (e.g., transcription, DNA replication) [11-14, $59,69,70]$, as major determinants for cohesin distribution in vivo. Indeed, the changes in cohesin distribution and accumulation after histone depletion might be due to defects in transcription and/or replication fork stability, as these processes are severely impaired in histonedepleted cells $[71,72]$.

\section{Conclusions}

In summary, our study has explored some of the connections between cohesin activity, chromatin integrity and transcription. We show that the binding and distribution of the cohesin peaks do not require a precise nucleosome organization, whereas chromatin integrity relies on cohesin activity at some regions, including promoters. These observations, together with the association of the cohesin-regulated genes with specific promoter chromatin architecture, suggest a role for chromatin dynamics in the regulation of transcription by cohesins. Remarkably, OPN genes (and more specifically SAGA-dominated genes) are characterized by high transcriptional plasticity $[37,43]$, which is a requirement for genes that control development, proliferation and pluripotency. In humans, the genes with the highest nucleosome occupancy at the TSS-proximal region (OPN-like genes) display high transcriptional plasticity [37]. It will therefore be important to study the chromatin structure of cohesin-regulated promoters in humans, as well as the impact that cohesins may have on the more complex human chromatin structure. This may be particularly relevant to understanding cohesinopathies, such as Cornelia de Lange syndrome and Roberts syndrome, which are associated with cohesin mutants that are proficient in cohesion but defective in gene expression [73].

\section{Methods}

Yeast strains, plasmids and growth conditions

Yeast strains used in this study are listed in Additional file 7: Table S7a. Tagged strains and deletion mutants were constructed by a PCR-based strategy [74]. For G1 synchronization, cells were grown in supplemented minimal medium (SMM) to mid-log-phase and $\alpha$ factor was 
added twice at 90-min intervals at $0.5 \mu \mathrm{g} / \mathrm{ml}$, except for $t:: H H F 2$ strains, which were treated with $1 \mu \mathrm{g} / \mathrm{ml}$. Cells were then washed three times and released into fresh SMM with $50 \mu \mathrm{g} / \mathrm{ml}$ pronase, except for cells released in the presence of nocodazole, which were released in rich medium (YPAD). To induce nucleosome depletion, $t:: H H F 2$ cells growing in the presence of $5 \mu \mathrm{g} / \mathrm{ml}$ doxycycline were shifted to $0.25 \mu \mathrm{g} / \mathrm{ml}$ during G1 synchronization and release. Cell cycle progression was followed by flow cytometry, budding index and DAPI staining.

\section{Western blot}

Protein extracts were obtained from cell cultures treated with $0.1 \mathrm{M}$ sodium azide to stop cell growth. Briefly, $25 \mathrm{ml}$ cultures were incubated in ice-cold 0.1 Tris- $\mathrm{HCl} \mathrm{pH} \mathrm{9.4,}$ $10 \mathrm{mM}$ DTT solution for $15 \mathrm{~min}$, collected by centrifugation, washed with $20 \mathrm{mM}$ HEPES pH 7.4, 1.2 M sorbitol, $1 \times$ cocktail inhibitor (Roche), and incubated in the same solution with $0.21 \mathrm{mg}$ of zymolyase $20 \mathrm{~T}$ for $1 \mathrm{~h}$ at $30{ }^{\circ} \mathrm{C}$. Spheroplasts were then washed twice with $20 \mathrm{mM}$ Tris$\mathrm{HCl} \mathrm{pH} \mathrm{7.5,} 20 \mathrm{mM} \mathrm{KCl}, 1 \mathrm{M}$ sorbitol, $1 \times$ cocktail inhibitor, $0.1 \mu \mathrm{M}$ spermidine and $0.25 \mu \mathrm{M}$ spermine and lysed with cold $20 \mathrm{mM}$ Tris- $\mathrm{HCl} \mathrm{pH} \mathrm{7.5,} 20 \mathrm{mM} \mathrm{KCl,} \mathrm{0.4} \mathrm{M}$ sorbitol, $1 \%$ Triton, $1 \times$ cocktail inhibitor, $0.1 \mu \mathrm{M}$ spermidine and $0.25 \mu \mathrm{M}$ spermine solution for $5 \mathrm{~min}$. Lysed extracts were mixed with Laemmli buffer, boiled and run on an SDS-polyacrylamide gel. Proteins were transferred to a nitrocellulose membrane (Hybond-ECL) that was blocked in PBS-T milk 5\% and incubated with primary antibodies against HA (rat monoclonal 3F10, Roche), Pgk1 (mouse polyclonal 22C5D8, Invitrogen), or histone H4 (rabbit polyclonal ab10158, Abcam). Proteins were detected using a peroxidase-conjugated goat anti-mouse or anti-rabbit IgG (both from Bio-Rad) secondary antibody and the ChemiDoc Gel Imaging System.

\section{Chromatin analysis by MNasel digestion and indirect end labeling}

G2/M cells were fixed for 15 min with $1 \%$ formaldehyde. Glycine was added to quench the reaction at a final concentration of $125 \mathrm{mM}$. Cells were sedimented, washed twice with cold TBS and stored at $-80{ }^{\circ} \mathrm{C}$ until use. Extracts for MNase digestion were re-suspended in $1 \mathrm{M}$ sorbitol and digested $1 \mathrm{~h}$ with $4.5 \mathrm{mg}$ of zymolyase 20T (AmsBio 120491-1). Samples were washed first with $1 \mathrm{M}$ sorbitol and then with $1 \mathrm{M}$ sorbitol $0.1 \mathrm{mM}$ PMSF, suspended gently in solution II (20 mM Tris$\mathrm{HCl}, 2 \mathrm{mM}$ EDTA, $0.15 \mathrm{M} \mathrm{NaCl}, 0.1 \mathrm{mM}$ PMSF, $0.2 \%$ Triton), and treated $30 \mathrm{~min}$ with different concentrations of MNase (SIGMA N3755). The reaction was then stopped by adding $0.4 \%$ SDS, $8.5 \mathrm{mM}$ EDTA. To revert cross-linking, samples were incubated for $90 \mathrm{~min}$ at $37^{\circ} \mathrm{C}$ with proteinase $\mathrm{K}$ and then overnight at $65^{\circ} \mathrm{C}$. DNA was extracted from samples using a standard phenol-chloroform extraction, treated with RNase A and loaded in a $1 \%$ agarose gel to check MNase digestion. MNase digestions used for indirect end labeling were incubated with the indicated restriction enzymes, resolved in 1.5\% agarose gels, blotted onto a HybondTM-XL membrane and probed with $\sim 200$ to $250 \mathrm{bp}{ }^{32} \mathrm{P}$-labeled specific PCR fragments. These fragments were always located close to one of the two ends of the fragment analyzed. Oligonucleotides for PCR amplification are listed in Additional file 7: Table S7b. Signals were acquired in a Fuji FLA5100 with the Image Gauge analysis program.

\section{Chromatin analysis by MNase-seq}

MNaseI-digested DNA samples from two biological replicates for each yeast strain were obtained as previously indicated for indirect end labeling. MNase-digested samples enriched in mononucleosomes were loaded in a $1 \%$ agarose gel, and the DNA corresponding to mononucleosomes was purified with a DNA purification kit (Qiagen). The DNA size and quality were confirmed by an electropherogram analysis (2100 Bioanalyzer ${ }^{\mathrm{TM}}$ ) (Additional file 10: Fig. S3). Library construction and sequencing was performed at Genomics Core Facility of CABIMER. DNA libraries were prepared from $100 \mathrm{ng}$ mononucleosome DNA using the Ion Plus Fragment Library Kit (Thermo Fisher), and the size distribution and molarity of each library were analyzed with the Agilent ${ }^{\mathrm{TM}}$ DNA High Sensitivity Kit (Agilent 2100 Bioanalyzer). DNA libraries were sequenced on the Ion Torrent ${ }^{\mathrm{TM}}$ Personal Genome Machine ${ }^{\mathrm{TM}}$ (PGM), and raw data were processed for base calling, filtering and trimming to generate the FASTQ files using the Torrent Suite ${ }^{\mathrm{TM}}$ Software. Sequence reads were mapped to $S$. cerevisiae genome sacCer3 by BowTie2 [75], and potential PCR duplicates were removed by SAM Tools on the Galaxy platform (usegalaxy.org) [76]. The peak-calling algorithm Dpos function (DANPOS 2.2.0) [35, 77] was used for nucleosome occupancy maps and comparative analyses using default parameters. Dynamic nucleosomes were selected using a point_diff_log10pval >-15, and they were classified into three categories: position shift (range setting between 50 and $90 \mathrm{bp}$ ), fuzziness (fuzziness_diff_log10pval >-15) and occupancy changes (smt_diff_log10pval $>-15$ ). Average nucleosome distance, occupancy and fuzziness were analyzed and plotted with the stat function (DANPOS). Average nucleosome occupancy patterns flanking transcription start sites (TSS) were plotted in either average density or heat maps using Profiles function (DANPOS) or DeepTools (usegalaxy.org), respectively [78]. 


\section{Chromatin immunoprecipitation (ChIP)}

Cells in the indicated conditions were fixed for $15 \mathrm{~min}$ with $1 \%$ formaldehyde. Glycine was added to quench the reaction at a final concentration of $125 \mathrm{mM}$. Cells were sedimented, washed twice with cold TBS and stored at $-80{ }^{\circ} \mathrm{C}$ until use. ChIP samples were obtained by breaking cells with a homogenizer (Multibeads shocker, Yasui Kikai) for $1 \mathrm{~h}$ at $2500 \mathrm{rpm}$ (30-s on/30-s off intervals) in lysis buffer (50 mM HEPES, $140 \mathrm{mM} \mathrm{NaCl}, 1 \mathrm{mM}$ EDTA, $1 \%$ Triton, $0.1 \%$ sodium deoxycholate, $1 \mathrm{mM}$ PMSF) supplemented with protease cocktail inhibitors (Roche). Supernatant was transferred to new tubes by soft centrifugation piercing at the bottom of the tube with a G25 needle, and chromatin was further concentrated by centrifugation. Chromatin was sheared via sonication to a size between 200 and 600 bp using a sonicator (Branson Digital Sonifier, Branson Ultrasonics). About $10 \mu \mathrm{l}$ of supernatant was kept on ice and used as the input DNA control; the rest was incubated overnight with $1 \mu \mathrm{g}$ of antibody at $4{ }^{\circ} \mathrm{C}$. Antibodies used include IgG (A4416, Sigma), anti-HA (3F10, Roche), anti-H3K56Ac (39281, Active Motif) and anti-H3 (AB1791, Abcam). All samples were immunoprecipitated using magnetic Protein G Dynabeads (Invitrogen; 10003D) for $90 \mathrm{~min}$. After immunoprecipitation, samples were washed twice with $1 \mathrm{ml}$ of the following solutions: lysis buffer, lysis buffer plus $0.5 \mathrm{M}$ $\mathrm{NaCl}$, wash buffer $(0.25 \mathrm{M} \mathrm{LiCl}, 10 \mathrm{mM}$ Tris- $\mathrm{HCl}, 1 \mathrm{mM}$ EDTA, $0.5 \%$ NP-40, 0.5\% sodium deoxycholate) and TE $1 \times$. Samples were then eluted from magnetic beads with a $1 \%$ SDS TE solution, incubated overnight at $65{ }^{\circ} \mathrm{C}$ to reverse cross-linking, treated with $0.15 \mathrm{mg}$ of proteinase $\mathrm{K}$ and extracted using a standard phenol/chloroform DNA purification. ChIP data were obtained by realtime qPCR using SYBR Green Premix Ex Taq (Takara). In Fig. 5e, IGRs $1-3$ contained the promoters of ERR2, IWR 1 and GAT3, and tDNA 1 and 2 are tP(UGG)N1 and tK(CUU)E1 (see Additional file 1: Fig. S7b for primers).

\section{Cohesin binding by ChIP-on-chip}

High-resolution (5-bp) ChIP-on-chip analyses were performed with two biological samples for wild type and $t:: H H F 2$. In each case, input (I) and immunoprecipitated (IP) DNA with antibody against HA from HA-Scc1 and untagged cells grown under the indicated conditions were obtained by ChIP (as described above) and then additionally purified through a DNA purification column (Qiagen). DNA was amplified by random priming using a GenomePlex $^{\circledR}$ Whole Genome Amplification (WGA) kit and cleaned with GenElute ${ }^{\mathrm{TM}}$ PCR Clean-Up Kit (SigmaAldrich). A total of $7.5 \mu \mathrm{g}$ of amplified DNA was fragmented, labeled and hybridized with the GeneChip $S$. cerevisiae Tiling 1.0R array (Affymetrix Inc.) following the manufacturer's procedure (25-bp probes shifted every $5 \mathrm{bp}$ ). ChIP-on-chip data were analyzed using the Tiling Array Suite 1.1.02 (TAS) software from Affymetrix. TAS produces the signal $(\log 2$ ratio IP/I) and the $p$ value intensity files per probe position (150 bp bandwidth around the inspected probe using quantile normalization plus scaling). Positive enrichment intervals were filtered as the regions with $p$ value $<0.05$ and a positive signal [(IP/I) - (IP/I) untag], considering a maximum gap of $250 \mathrm{bp}$ and a minimum run/length of $30 \mathrm{bp}$.

\section{Transcription analysis by RNA-seq}

RNA was extracted using a standard hot acid phenol extraction protocol [79] and purified with the RNeasy Mini kit (Qiagen). RNA samples were analyzed for quality with the RNA 6000 Nano assay on a 2100 Bioanalyzer (Agilent Technologies) and quantified with the Qubit ${ }^{\mathrm{TM}}$ RNA HS Assay (Thermo Fisher Scientific ${ }^{\mathrm{TM}}$ ). RNA libraries from two independent biological replicates for wild type and scc1-73 were constructed and sequenced at the Genomics Core Facility of CABIMER. For this, strandspecific total RNA-seq libraries from 100 ng RNA samples were prepared using the TruSeq ${ }^{\circledR}$ Stranded mRNA Library prep kit (Illumina). Indexed libraries were pooled and sequenced on Illumina NextSeq 500 using paired-end chemistry with $75 \mathrm{bp}$ read length to a depth of approximately about 37 million reads per library. Raw reads were filtered and trimmed with FASTQ toolkit 1.0.0 and assessed using FastQC 1.0.0 by BaseSpace Sequence Hub Illumina website. FASTQ data were uploaded to the Galaxy web platform for further analyses [76]. Briefly, reads were aligned to the $S$. cerevisiae genome version sacCer3 using the HISAT2 aligner [80] and filtered for high-quality mapping (MapQuality $\geq 30$ ) using BAM tools [81]. Differential expression analyses including fold change and statistical significance of gene expression profiles were performed using the Cuffdiff program [82] and the reference annotation sacCer3.gtf (downloaded from UCSC). Gene ontology analyses were performed with the DAVID Bioinformatics resources [83].

\section{Genome-wide data}

Nucleosome profiles and cohesin peaks along the genome were visualized using the Integrative Genomics Viewer (IGV) [84]. All data are MIAME compliant. Raw data have been deposited at the MIAME-compliant Gene Expression Omnibus (GEO) database at the National Center for Biotechnology Information (http://www.ncbi. nlm.nih.gov/geo/) and are accessible through the accession numbers GSE121067 (MNaseI-seq), GSE121004 (ChIP-on-chip) and GSE125258 (RNA-seq). 


\section{Comparative analyses of genomic elements} Analyses of the genome distribution and overlapping of dynamic nucleosomes, cohesin peaks and gene elements were performed with the tools of the Galaxy web platform (usegalaxy.org) [76]. Other statistical analyses were performed with the GraphPad Prism software.

\section{Additional files}

Additional file 1: Table S1. Comparison of the number and percentage of nucleosomes altered after histone depletion (in t::HHF2 cells) or cohesin inactivation (in scc1-73 cells) in the indicated genomic regions relative to the whole genome. A hypergeometric test was used to determine the probability to obtain the indicated percentages of altered nucleosomes at the indicated genomic regions if their distribution along the genome were random. Significantly higher (green) and lower (red) percentages are highlighted. *rDNA is analyzed as a single copy.

Additional file 2: Table S2. Comparison of the number and percentage of nucleosomes altered after histone depletion (in $t::$ HHF2 cells) or cohesin inactivation (in scc1-73 cells) in the indicated genomic regions relative to the whole genome. A hypergeometric test was used to determine the probability of obtaining the indicated percentages of altered nucleosomes at the indicated genomic regions if their distribution along the genome were random. Significantly higher (green) and lower (red) percentages are highlighted. ${ }^{*}$ rDNA, analyzed as a single copy.

Additional file 3: Table S3. Misregulated genes in ssc1-73. Lists of up- and downregulated genes are shown.

Additional file 4: Table S4 List of misregulated genes from additional studies used for comparative analyses in Table 2.

Additional file 5: Table S5. Cohesin distribution in wild-type and histonedepleted cells at the indicated genomic regions. A peak of Scc1 is defined as a DNA fragment with continuous $\mathrm{Scc} 1$ signals that are both positive (relative to the untagged strain) and with a $p<0.05$. A peak signal was calculated as the sum of these positive signals. Genomic regions with Scc1 and peaks of $\mathrm{Scc} 1$ at a particular genomic region are defined by at least 1 bp overlapping.

Additional file 6: Table S6. The effect of cohesin inactivation on chromatin structure is more severe in cohesin-associated regions. The probability that scc1-73 alters nucleosomes in Scc1-binding IGR or Scc1-binding ORF if the altered genomic regions in scc $1-73$ cells were randomly distributed was determined by a hypergeometric test. The rate between the observed and expected frequencies of common genomic regions is also shown. The number of regions in each case is indicated in parenthesis.

Additional file 7: Table S7. Saccharomyces cerevisiae strains (a) and oligos (b) used in this study.

Additional file 8: Fig. S1. Effect of cohesin inactivation in scc1-73 cells on the chromatin structure of three different loci of histone-depleted cells. Nucleosome positioning analyses are shown for the indicated loci after MNase I digestion and indirect-end labeling of the indicated strains synchronized in $\mathrm{G} 1$ and released into fresh medium for $1 \mathrm{~h}$ at $37^{\circ} \mathrm{C}$ until G2/M.

Additional file 9: Fig. S2. Effect of histone depletion on cohesin binding and distribution. a Cohesin distribution at different regions of chromosomes $I I$ and $V$ in wild-type and t::HHF2 cells that have been synchronized in $\mathrm{G} 1$ and released into fresh medium until G2/M, as determined by ChIPon-chip analysis against HA-Scc1. b Probability that IGR that had either lost or gained cohesins after histone depletion would overlap with IGR with altered nucleosomes in t::HHF2 cells or in both $t::$ HHF2 and scc1-73 cells if they were randomly distributed, as determined by a hypergeometric test. The rate between the observed and expected frequencies of common IGR is also shown. The number of IGR in each case is indicated in parenthesis. nd, not determined.

Additional file 10: Fig. S3. Preparation and analysis of the nucleosomal DNA used for MNasel-seq. a Generation of mononucleosomes in the indicated strains after partial digestions with MNase I. The DNA purified for DNA-seq is marked in red. $\mathbf{b}, \mathbf{c}$ Electrophoretic (b) and electropherogram (c) analyses of the purified nucleosomal DNA.

\section{Abbreviations}

ARS: autonomous replication sequence; DANPOS: dynamic analysis of nucleosome position and occupancy by sequencing; DPN: depleted-proximal promoter; FN: fragile nucleosome; IGR: intergenic region; NFR: nucleosome-free region; OPN: occupied-proximal promoter; ORF: open reading frame; rDNA: ribosomal DNA; SN: stable nucleosome; tDNA: transfer DNA; TSS: transcription start site.

\section{Acknowledgements}

We thank José Carlos Reyes for critically reading the manuscript.

\section{Authors' contributions}

DM, MM-P, MB-M, MJC-L, EG-M and MM-H performed the experiments. DM, EA, MP-A, MM-P, MB-M and FP analyzed the results. FP conceived the project and wrote the manuscript. All authors read and approved the final manuscript.

\section{Funding}

This work was supported by the Spanish Ministry of Economy and Competitiveness (BFU2012-38171 and BFU2015-63698-P) and the Andalusian Government (P12-CTS-2270).

\section{Availability of data and materials}

Genome-wide data have been deposited at the MIAME-compliant Gene Expression Omnibus (GEO). The remaining data and material are available from the corresponding author on request.

\section{Ethics approval and consent to participate}

Not applicable.

\section{Consent for publication}

Not applicable.

\section{Competing interests}

The authors declare that they have no competing interests.

\section{Author details}

${ }^{1}$ Department of Genome Biology, Andalusian Molecular Biology and Regenerative Medicine (CABIMER), CSIC-University of Seville-University Pablo de Olavide, Seville, Spain. ${ }^{2}$ Genomic Unit, Andalusian Molecular Biology and Regenerative Medicine Center (CABIMER), CSIC-University of Seville-University Pablo de Olavide, Seville, Spain. ${ }^{3}$ Present Address: Department of Biochemistry, University of Oxford, Oxford, UK. ${ }^{4}$ Present Address: Division of Cancer Sciences, Manchester Cancer Research Center, University of Manchester, Manchester, UK.

Received: 22 March 2019 Accepted: 10 July 2019

Published online: 22 July 2019

\section{References}

1. Prado F, Maya D. Regulation of replication fork advance and stability by nucleosome assembly. Genes (Basel). 2017;8:49.

2. Polo SE, Almouzni G. Chromatin dynamics after DNA damage: the legacy of the access-repair-restore model. DNA Repair. 2015;36:114-21.

3. Venkatesh S, Workman JL. Histone exchange, chromatin structure and the regulation of transcription. Nature. 2015;16:178-89.

4. Maeshima K, Ide S, Babokhov M. Dynamic chromatin organization without the 30-nm fiber. Curr Opin Cell Biol. 2019;58:95-104. 
5. Kschonsak M, Haering CH. Shaping mitotic chromosomes: from classical concepts to molecular mechanisms. BioEssays. 2015;37:755-66.

6. Nitiss JL. DNA topoisomerase II and its growing repertoire of biological functions. Nat Rev Cancer. 2009;9:327-37.

7. Kakui Y, Uhlmann F. SMC complexes orchestrate the mitotic chromatin interaction landscape. Curr Genet. 2017;64:335-9.

8. Michaelis C, Ciosk R, Nasmyth K. Cohesins: chromosomal proteins that prevent premature separation of sister chromatids. Cell. 1997;91:35-45.

9. Ciosk R, Shirayama M, Shevchenko A, Tanaka T, Toth A, Shevchenko A, et al. Cohesin's binding to chromosomes depends on a separate complex consisting of Scc2 and Scc4 proteins. Mol Cell. 2000;5:243-54.

10. Srinivasan M, Scheinost JC, Petela NJ, Gligoris TG, Wissler M, Ogushi S, et al. The cohesin ring uses its hinge to organize DNA using non-topological as well as topological mechanisms. Cell. 2018;173:1-31.

11. Glynn EF, Megee PC, Yu H-G, Mistrot C, Ünal E, Koshland DE, et al. Genome-wide mapping of the cohesin complex in the yeast Saccharomyces cerevisiae. PLoS Biol. 2004;2:e259-15.

12. Lengronne A, Katou Y, Mori S, Yokobayashi S, Kelly GP, Itoh T, et al. Cohesin relocation from sites of chromosomal loading to places of convergent transcription. Nature. 2004:430:573-8.

13. Laloraya S, Guacci V, Koshland D. Chromosomal addresses of the cohesin component Mcd1 p. J Cell Biol. 2000;151:1047-56.

14. Stigler J, Çamdere GÖ, Koshland DE, Greene EC. Single-molecule imaging reveals a collapsed conformational state for DNA-bound cohesin. Cell Rep. 2016;15:988-98.

15. Tóth A, Ciosk R, Uhlmann F, Galova M, Schleiffer A, Nasmyth K. Yeast cohesin complex requires a conserved protein, Eco 1 p(Ctf7), to establish cohesion between sister chromatids during DNA replication. Genes Dev. 1999;13:320-33.

16. Lengronne A, Mclntyre J, Katou Y, Kanoh Y, Hopfner K-P, Shirahige K, et al. Establishment of sister chromatid cohesion at the $S$. cerevisiae replication fork. Mol Cell. 2006;23:787-99.

17. Murayama Y, Samora CP, Kurokawa Y, Iwasaki H, UhImann F. Establishment of DNA-DNA interactions by the cohesin ring. Cell. 2018:172(465-469):e15.

18. Rhodes JDP, Haarhuis JHI, Grimm JB, Rowland BD, Lavis LD, Nasmyth KA. Cohesin can remain associated with chromosomes during DNA replication. Cell Rep. 2017;20:2749-55.

19. Uhlmann F, Lottspeich F, Nasmyth K. Sister-chromatid separation at anaphase onset is promoted by cleavage of the cohesin subunit Scc1. Nature. 1999:400:37-42.

20. Makrantoni V, Marston AL. Cohesin and chromosome segregation. Curr Biol. 2018;28:R688-93.

21. Uhlmann F. SMC complexes: from DNA to chromosomes. Nature. 2016;17:1-14

22. Villa-Hernández $\mathrm{S}$, Bermejo R. Cohesin dynamic association to chromatin and interfacing with replication forks in genome integrity maintenance. Curr Genet. 2018;16:281.

23. Remeseiro S, Losada A. Cohesin, a chromatin engagement ring. Curr Opin Cell Biol. 2013;25:63-71.

24. Dorsett D, Merkenschlager M. Cohesin at active genes: a unifying theme for cohesin and gene expression from model organisms to humans. Curr Opin Cell Biol. 2013;25:327-33.

25. Mehta GD, Kumar R, Srivastava S, Ghosh SK. Cohesin: functions beyond sister chromatid cohesion. FEBS Lett. 2013;587:2299-312.

26. Lopez-Serra L, Kelly G, Patel H, Stewart A, Uhlmann F. The Scc2-Scc4 complex acts in sister chromatid cohesion and transcriptional regulation by maintaining nucleosome-free regions. Nat Genet. 2014;46:1147-51.

27. Huang J, Hsu J-M, Laurent BC. The RSC nucleosome-remodeling complex is required for cohesin's association with chromosome arms. Mol Cell. 2004;13:739-50.

28. Muñoz S, Minamino M, Casas-Delucchi CS, Patel H, Uhlmann F. A role for chromatin remodeling in cohesin loading onto chromosomes. Mol Cell. 2019;74:664-5.

29. Litwin I, Bakowski T, Maciaszczyk-Dziubinska E, Wysocki R. The LSH/HELLS homolog Irc5 contributes to cohesin association with chromatin in yeast. Nucl Acids Res. 2017;45:6404-16.

30. Hakimi M-A, Bochar DA, Schmiesing JA, Dong Y, Barak OG, Speicher DW, et al. A chromatin remodelling complex that loads cohesin onto human chromosomes. Nature. 2002;418:994-8.
31. Liu J, Czajkowsky DM, Liang S, Shao Z. Cell cycle-dependent nucleosome occupancy at cohesin binding sites in yeast chromosomes. Genomics. 2008;91:274-80.

32. Blat Y, Kleckner N. Cohesins bind to preferential sites along yeast chromosome III, with differential regulation along arms versus the centric region. Cell. 1999;98:249-59.

33. Lee C-K, Shibata Y, Rao B, Strahl BD, Lieb JD. Evidence for nucleosome depletion at active regulatory regions genome-wide. Nat Genet. 2004:36:900-5.

34. Yuan G-C, Liu Y-J, Dion MF, Slack MD, Wu LF, Altschuler SJ, et al. Genomescale identification of nucleosome positions in S. cerevisiae. Science. 2005:309:626-30.

35. Chen $K, X i Y$, Pan X, Li Z, Kaestner K, Tyler J, et al. DANPOS: dynamic analysis of nucleosome position and occupancy by sequencing. Genome Res. 2013;23:341-51.

36. Haering CH, Schoffnegger D, Nishino T, Helmhart W, Nasmyth K, Löwe J. Structure and stability of cohesin's Smc1-Kleisin interaction. Mol Cell. 2004;15:951-64.

37. Tirosh I, Barkai N. Two strategies for gene regulation by promoter nucleosomes. Genome Res. 2008;18:1084-91.

38. Zaugg JB, Luscombe NM. A genomic model of condition-specific nucleosome behavior explains transcriptional activity in yeast. Genome Res. 2012;22:84-94

39. Kubik S, Bruzzone MJ, Jacquet P, Falcone J-L, Rougemont J, Shore D. Nucleosome stability distinguishes two different promoter types at al protein-coding genes in yeast. Mol Cell. 2015;60:422-34.

40. Kubik S, Bruzzone MJ, Shore D. Establishing nucleosome architecture and stability at promoters: roles of pioneer transcription factors and the RSC chromatin remodeler. BioEssays. 2017;39:1600237-10.

41. Floer M, Wang X, Prabhu V, Berrozpe G, Narayan S, Spagna D, et al. A RSC/ nucleosome complex determines chromatin architecture and facilitates activator binding. Cell. 2010;141:1-12.

42. Basehoar AD, Zanton SJ, Pugh BF. Identification and distinct regulation of yeast TATA box-containing genes. Cell. 2004;1 16:699-709.

43. Huisinga KL, Pugh BF. A genome-wide housekeeping role for TFIID and a highly regulated stress-related role for SAGA in Saccharomyces cerevisiae. Mol Cell. 2004;13:573-85.

44. Petrenko N, Jin Y, Wong KH, Struhl K. Evidence that Mediator is essential for Pol II transcription, but is not a required component of the preinitiation complex in vivo. eLife. 2017;6:e28447.

45. Bruzzone MJ, Grünberg S, Kubik S, Zentner GE, Shore D. Distinct patterns of histone acetyltransferase and Mediator deployment at yeast proteincoding genes. Genes Dev. 2018;32:1252-65.

46. Morillo-Huesca M, Clemente-Ruiz M, Andújar E, Prado F. The SWR1 histone replacement complex causes genetic instability and genomewide transcription misregulation in the absence of H2A.Z. PLOS ONE. 2010:5:e12143.

47. Murillo-Pineda M, Cabello-Lobato MJ, Clemente-Ruiz M, Monje-Casas F, Prado F. Defective histone supply causes condensin-dependent chromatin alterations, SAC activation and chromosome decatenation impairment. Nuclic Acids Res. 2014;42:12469-82. Corrigendum: Nucl Acids Res. 2016. https://doi.org/10.1093/nar/gkw058.

48. Prado F, Aguilera A. Partial depletion of histone $\mathrm{H} 4$ increases homologous recombination-mediated genetic instability. Mol Cell Biol. 2005:25:1526-36.

49. Gossett AJ, Lieb JD. In vivo effects of histone H3 depletion on nucleosome occupancy and position in Saccharomyces cerevisiae. PLoS Genet. 2012:8:e1002771.

50. Eaton ML, Galani K, Kang S, Bell SP, MacAlpine DM. Conserved nucleosome positioning defines replication origins. Genes Dev. 2010;24:748-53.

51. Liu S, Xu Z, Leng H, Zheng P, Yang J, Chen K, et al. RPA binds histone $\mathrm{H} 3-\mathrm{H} 4$ and functions in DNA replication-coupled nucleosome assembly. Science. 2017;355:415-20.

52. Barlow T, Eliasson R, Platz A, Reichard P, Sjöberg BM. Enzymic modification of a tyrosine residue to a stable free radical in ribonucleotide reductase. Proc Natl Acad Sci USA. 1983;80:1492-5.

53. Hu B, Petela N, Kurze A, Chan KL, Chapard C, Nasmyth K. Biological chromodynamics: a general method for measuring protein occupancy across the genome by calibrating ChIP-seq. Nucl Acids Res. 2015;43:e132. 
54. Brackley CA, Allan J, Keszenman-Pereyra D, Marenduzzo D. Topological constraints strongly affect chromatin reconstitution in silico. Nucl Acids Res. 2014;43:63-73.

55. Rao SSP, Huang S-C, Hilaire BGS, Engreitz JM, Perez EM, Kieffer-Kwon K-R et al. Cohesin loss eliminates all loop domains. Cell. 2017;171:305-24.

56. Schalbetter SA, Goloborodko A, Fudenberg G, Belton J-M, Miles C, Yu $M$, et al. SMC complexes differentially compact mitotic chromosomes according to genomic context. Nat Cell Biol. 2017;19:1071-80.

57. Lazar Stefanita L, Scolari VF, Mercy G, Muller H, Guérin TM, Thierry A, et al. Cohesins and condensins orchestrate the 4D dynamics of yeast chromosomes during the cell cycle. EMBO J. 2017;36:2684-97.

58. Frattini C, Villa-Hernández S, Pellicanò G, Jossen R, Katou Y, Shirahige K, et al. Cohesin ubiquitylation and mobilization facilitate stalled replication fork dynamics. Mol Cell. 2017;68(758-771):e5.

59. Samora CP, Saksouk J, Goswami P, Wade Ben O, Singleton MR, Bates PA, et al. Ctf4 Links DNA replication with sister chromatid cohesion establishment by recruiting the Chl1 helicase to the replisome. Mol Cell. 2016;63:1-33.

60. Huang H, Strømme CB, Saredi G, Hödl M, Strandsby A, González-Aguilera $C$, et al. A unique binding mode enables MCM2 to chaperone histones H3-H4 at replication forks. Nat Struct Mol Biol. 2015;22:618-26.

61. Foltman M, Evrin C, De Piccoli G, Jones RC, Edmondson RD, Katou Y, et al. Eukaryotic replisome components cooperate to process histones during chromosome replication. Cell Rep. 2013;3:892-904.

62. Dorsett D. Cohesin: genomic insights into controlling gene transcription and development. Curr Opin Genet Dev. 2011;21:199-206.

63. Lenstra TL, Benschop JJ, Kim T, Schulze JM, Brabers NACH, Margaritis T, et al. The specificity and topology of chromatin interaction pathways in yeast. Mol Cell. 2011:42:536-49.

64. van Ruiten MS, Rowland BD. SMC complexes: universal DNA looping machines with distinct regulators. Trends Genet. 2018;34:477-87.

65. Chereji RV, Ocampo J, Clark DJ. MNase-sensitive complexes in yeast: nucleosomes and non-histone barriers. Mol Cell. 2017:65(565-577):e3.

66. Brahma S, Henikoff S. RSC-associated subnucleosomes define MNasesensitive promoters in yeast. Mol Cell. 2019;73(238-249):e3.

67. Kubik S, O'Duibhir E, de Jonge WJ, Mattarocci S, Albert B, Falcone J-L, et al. Sequence-directed action of RSC remodeler and general regulatory factors modulates +1 nucleosome position to facilitate transcription. Mol Cell. 2018;71(89-102):e5.

68. Sun M, Nishino T, Marko JF. The SMC1-SMC3 cohesin heterodimer struc tures DNA through supercoiling-dependent loop formation. Nucl Acids Res. 2013:41:6149-60.

69. Xu H, Boone C, Brown GW. Genetic dissection of parallel sister-chromatid cohesion pathways. Genetics. 2007;176:1417-29.

70. Borges V, Smith DJ, Whitehouse I, Uhlmann F. An Eco1-independent sister chromatid cohesion establishment pathway in S. cerevisiae. Chromosoma. 2013;122:121-34
71. Clemente-Ruiz M, Prado F. Chromatin assembly controls replication fork stability. EMBO Rep. 2009;10:790-6.

72. Wyrick JJ, Holstege FC, Jennings EG, Causton HC, Shore D, Grunstein M, et al. Chromosomal landscape of nucleosome-dependent gene expression and silencing in yeast. Nature. 1999;402:418-21.

73. Remeseiro S, Cuadrado A, Losada A. Cohesin in development and disease. Development. 2013;140:3715-8.

74. Longtine MS, Mckenzie A III, Demarini DJ, Shah NG, Wach A, Brachat A, et al. Additional modules for versatile and economical PCR-based gene deletion and modification in Saccharomyces cerevisiae. Yeast. 1998;14:953-61.

75. Langmead Ben, Salzberg SL. Fast gapped-read alignment with Bowtie 2 . Nat Methods. 2012;9:357-9.

76. Afgan E, Baker D, van den Beek M, Blankenberg D, Bouvier D, Čech M, et al. The Galaxy platform for accessible, reproducible and collaborative biomedical analyses: 2016 update. Nucl Acids Res. 2016;44:W3-10.

77. Chen K, Chen Z, Wu D, Zhang L, Lin X, Su J, et al. Broad H3K4me3 is associated with increased transcription elongation and enhancer activity at tumor-suppressor genes. Nat Genet. 2015;47:1149-57.

78. Ramírez F, Ryan DP, Grüning B, Bhardwaj V, Kilpert F, Richter AS, et al. deepTools2: a next generation web server for deep-sequencing data analysis. Nucl Acids Res. 2016;44:W160-5.

79. Köhrer K, Domdey H. Preparation of high molecular weight RNA. Methods Enzymol. 1991;194:398-405.

80. Kim D, Langmead B, Salzberg SL. HISAT: a fast spliced aligner with low memory requirements. Nat Methods. 2015;12:357-60.

81. Barnett DW, Garrison EK, Quinlan AR, Strömberg MP, Marth GT. BamTools: a $\mathrm{C}++\mathrm{API}$ and toolkit for analyzing and managing BAM files. Bioinformatics. 2011;27:1691-2.

82. Trapnell C, Williams BA, Pertea G, Mortazavi A, Kwan G, van Baren MJ, et al. Transcript assembly and quantification by RNA-Seq reveals unannotated transcripts and isoform switching during cell differentiation. Nat Biotech. 2010;28:511-5.

83. Huang DW, Sherman BT, Lempicki RA. Systematic and integrative analysis of large gene lists using DAVID bioinformatics resources. Nat Protoc. 2009:4:44-57.

84. Robinson JT, Thorvaldsdóttir H, Winckler W, Guttman M, Lander ES, Getz G, et al. Integrative genomics viewer. Nat Biotechnol. 2011;29:24-6.

\section{Publisher's Note}

Springer Nature remains neutral with regard to jurisdictional claims in published maps and institutional affiliations.
Ready to submit your research? Choose BMC and benefit from:

- fast, convenient online submission

- thorough peer review by experienced researchers in your field

- rapid publication on acceptance

- support for research data, including large and complex data types

- gold Open Access which fosters wider collaboration and increased citations

- maximum visibility for your research: over $100 \mathrm{M}$ website views per year

At BMC, research is always in progress.

Learn more biomedcentral.com/submissions 